\title{
Sickness and Therapy Among Tribals of Rajasthan
}

\author{
Veena Bhasin
}

This paper describes the medical traditions that are being utilized by tribal groups namely Bhil, Mina, Garasia, Damor, Kathodi and Sahariya of Rajasthan for health problems. From the analysis of ethnographic data it was found that their medical sphere is highly pluralistic with emphasis on prevention, curative and promotive measures. Local medical traditions have continued to co-exist with biomedicine among tribals of this semi- arid zone, though it was widely assumed that biomedicine would ultimately replace the traditional medical practices. Biomedicine occupies an important place in the treatment of infirmity among tribals in these remoter parts due to the establishment of government sponsored health centers. Oral medicine and injections are popular and are administered by various unqualified as well as trained qualified practitioners. There are government sponsored Public Health Centres (PHCs), private medical practitioners practicing biomedicine as well as Ayurvedic medicine (herbal and mineral tradition of Indian Medicine). Beside biomedical and Ayurvedic practitioners there are number of traditional healers who cater to the needs of tribals. These are: -'specialists in home remedies; ritual care practitioners (bhopas) who also perform jhara- the ritual sweeping away of illness; herbalists (jaangar/ jaankar) who administer 'desi-dvai'; nabj or nadu (pulse) specialists; grain diviner (devala) who help in diagnosing disease; bonesetters; abdominal masseurs; priests (khoont) who use complainspecific mantras (sacred verbal formula); and midwives. The concept of dabna is also prevalent among tribals. Dabna is stamping on the affected part of the body with a hot iron piece. There are various patterns for different ailments. Dabna does not necessarily require the services of any specialist in case of human beings. However, in case of animals, services of experts are required. Along with, tribals believe in pilgrimage to temples, and healing at religious shrines by possessed mediums of a deity. Some practitioners are locally renowned for their expertise in treating certain ailments. Likewise some local deities are renowned for the management of specific problems. Most of the above mentioned specialists whether ritual or sacred have acquired their skills either through inheritance or through accidental detection of a fastidious gift from god or by training under the specialist of a particular medical tradition. The strength of these indigenous medical traditions is the fundamental knowledge or ethno medical concept that is shared by the whole community. Tribals of Rajasthan categorize different sets of conditions as illnesses of body and mind depending on the respective causes. Most of the illnesses by the symptoms of cold and fever are diseases of cold (sardi ki bimari); boils, spots, mouth ulcers, dath, heat stroke (lapat lagna) etc are diseases of heat (garmi ki bimari). Dietary precautions and folk remedies are used for treating wide range of such diseases. Regarding their health conditions the tribals are concerned with the causes of sickness and appropriate treatment. Apart from hot and cold illnesses, it is believed by tribals that sickness is caused by social offences against dead or living or celestial world. Spirits and ghosts cause various kinds of suffering and are agents of illness and fatality. Diseases are also believed to be caused by human intervention for example by a mooth, a voodoo type of ritual. The tribal quotes elaborate descriptions, incidents and examples to affirm the evil craft. Grains are an essential material of a mooth. This practice is mastered in cremation grounds or under a tree. It is said that extent of perfection achieved is tested by using the craft on trees. The effect produced is gradual wilting, drying and almost definite death of a completely blooming full sized green tree. The same fate would be met by a tribal on whom the mooth is blown by an enemy or a daken (witch) seeking vengeance. The tribals of Rajasthan believe that diseases can be transferred to other people by cross-road motif. The charms are kept on the crossings with the belief (i) that it will inflict harm to the person passing by or touching it accidentally; (ii) to appease an evil spirit or deity the believed cause of sufferance; and (iii) to leave affliction there. The charms consists of sweets, 
lighted dough lamps, vermilion, grains and lemons etc. Evil eye or nazar is considered an another cause of sickness.

The present paper focuses on health, sickness and therapy from the point of view of ordinary tribals of Rajasthan, real or prospective patients and their family members as well as local practitioners.

\section{THE CULTURAL CONTEXT OF HEALTH, DISEASE AND MEDICINE}

Every culture has its particular explanation for ill health. Culture provide people with ways of thinking, that are " simultaneously models of and models for reality"Geertz (1973) and Frake (1961) in the diagnosis of disease among Subanum of Mindannao, described sickness as a vehicle for pursuing other interests. The explanatory model (EM) of illness simultaneously creates order and meaning, gives plan for purposive action and helps to produce the conditions required for their own perpetuation or revision (Kleinman, 1980). While Kleinman and his associates have been establishing the EM approval, other anthropologists found that social relations primarily produce the forms and distribution of sickness in society.

Religion has been held responsible for many differences and norms affecting the fundamental values and behavioral pattern in life including health behavior. Every religion has three aspects: values, symbols and practices. People are forgetting the values but are struck with only the practices, symbols or customs. The religious beliefs of tribals are pluralistic, with both Hindu and Islamic religious perceptions being absorbed in their animistic religion. However, they consider themselves Hindu

The tribals believe in two main types of godsthe great Hindu gods whose place of worship is outside their village, and the village gods and house gods whose shrines are found within the village and in huts respectively. In addition, there are many spirits, such as ghosts of ancestors, hills the valleys, the darkness and trees, water places, river banks, old simple as well as step wells. All these places are moving grounds of ghosts, spirits and demons. It can be said that the popular religion of these tribals is demonolatry and in this there is no deep cleavage between the six tribal groups under the study. All of these gods, ghosts and spirits are capable of possessing people, animals and objects, and all have to be propitiated at one time or another with appropriate ceremonies. After sunset or on waning moon nights, or at midnights, the tribal are attentive in their movements, observing taboos and relying on their charms and amulets for the fear of encounter with the lurking super naturals, avoiding crossings, old and dilapidated structures, aged trees and those with richly fragrant flowers, cremation grounds and sites where burials might have taken place.

\section{TRADITIONAL EXPLANATION OF SICKNESS}

Accidents, calamities, diseases and losses are readily explained by holding some elements of supernatural or another responsible for. Among tribals the diseases that are not severe or life threatening are dealt with the specific therapies suggested after diagnosis. However in serious and socially disturbing ailments the prognostic dimensions come to the fore. In the course of diagnosis distinction between natural and supernatural causation is recognized. It is understood by tribals that a lot of sicknesses have natural causes and natural courses and are treated by traditional medical practitioners. These traditional practices focus not only on cure but on damage control measures as well to ensure speedy and uncomplicated recovery. Before starting any type of treatment one may tie charmed amulets for one cannot be always certain of physical etiology of the illness. Tying of a charmed amulet is common preliminary act which serves two functions (i) if the disease is caused by external agents, then the amulets may cure the disease. The charms also protect the individual against demonic interference. The efficacy of the amulets is generally for a limited period .If disease is not cured by amulet, a bigger ritual is required; (ii) an amulet may act as protection even if the cause is physical manifestation, for spirits can attack a person in physically weak state.

The pox diseases-small pox (barimata), measles (choti mata) and chicken pox (acparo) are attributed to the wrath of three mother goddesses, The manifestation of the pox disease is caused by heat of her presence within the human body. Tribals propitiate mother goddess by observing religious rituals and festivals. These propitiation rituals are well thought-out simple remedies. Tribals are aware that despite their worship their children may get inflicted with pox 
disease because of some mistake committed in ritual or worship. However, they believe that since they are observing all precautions, the disease will leave the body in a specific number of days with no permanent damage. It is believed by all tribals (lay men as well as folk healers ) that pox disease move vertically down through the body though the conceptual linkages between height, cleanliness, purity and ideational superiority that are directly articulated to human body and healing process are not understood by everybody. This gravitational, nonsymmetrical orientation is referred as process of "unmarking" (Marriott,1990). Diseases that are thought to originate in the head are expected to move downwards in a natural course from head to feet. Sometimes there is deviation in the natural course of illness i.e. it follows the opposite course and moves from feet to head. This 'ulta' (upside down) movement of sickness is considered dangerous and call for special diagnosis and subsequent treatment. The sicknesses which have a specified course and move in particular direction are time bound and are known as 'miyadi bimari.' The duration of such illnesses varies according to the severity and degree of indisposition. It varies from 7 to 21 days for different types. These types of prolonged sicknesses characterized by fever are referred as 'nikla' or 'came out'. An other sickness which is attributed to wrath of supernatural is 'Motijhara'(typhoid). It is believed by tribals that if 'Thakur Baba' is not worshipped at a proper time, his wrath may invoke 'Motijhara'. It is a condition caused by cold in the head accompanied by high fever. In its initial stages it is mild fever which moves downwards and starts appearing on chest. This initial condition is known as 'panijhara'. If this condition persists for longer period, it gets transformed in to 'Motijhara' which is characterised by the bad smell emitting from patient's body. For diagnosis local healers smell the palm of the patient's hand and look for 'spots'(Moti)) or rash on the chest. The appearance of rash and emanation of bad smell from body are considered a necessary and expected course of disease.

In cases of snakebite and scorpion bite the poisons move upwards. direction. Hence the treatment is directed towards preventing the poisonous fluids from reaching the head. It is not just the snake bite itself and the poisonous effect that is to be treated but also the shock as well. Therefore the treatment of snakebite requires special rituals as well. Generally the snake bite victims are carried to Goga ji than (shrine) at Gogameda near Ganganagar or to Kala kura bhagora of Kodia gaon in Dungarpur. The healer goes in to trance and invokes his deity. During trance he grabs Thuar(Euphorbia nerfolia) shrubs and starts biting and chewing its dendrons .After this he sucks venom out of the wound but does not vomit out the poison. The regurgitation of the mixture of latex rich pulp and poison from the snake bite annuls the poison effect. In some cases the folk healer perform only 'jhara' not accompanied by physical sucking of poison. The mere presence of ritual healer and his magical powers are sufficient to save the victim. This ritual tend to focus on symbolically encouraging and assisting the supposed course of sickness or on transferring it away from the patient's body instead of treatment in a precise way. As tribal frequently encounter snakes, even they are aware of the identity of poisonous and non- poisonous snakes. The persistent faith of tribal in 'jhara' may have the plausible explanation of autosuggestion or mesmerism.

Evil eye (nazar)is considered an other cause of sickness that afflicts persons who do not have strong resistance. Therapeutic rituals to counteract 'evil eye' effect require no esoteric knowledge. Certain diseases in young children are inferred to be the effect of 'evil eye' and this effect is removed by means of a vehicle of transference . In case of distended abdomen and reddened eyes of a child, Bhils give bath of crushed root bark of Kali doodhi (Ichnocarpus frutescens) dissolved in water to ward off the embodiment. The crushed roots of Kavach (Mucura pruriens) are administered to child victim of 'evil eye'. The abnormally distended stomach is reduced to normal following vomiting. This treatment is given to children aged four to six years with the belief that the embodiment of' 'evil eye' is vomited out. A common domestic remedy entails circling of a lemon sliced in to four quarters up to nearly $3 / 4$ of its vertical diameter with two strips of red cloth and vermilion passed diagonally through the cuts over the head of the sick child and then the lemon is kept in a clay pot and is set adrift in water after sealing the pot. In the absence of flowing water source, the pot is buried in the ground. Tribal leave charm of five red peppers, five crystals of salt and one lemon at sunset at the junction of four roads. All these practices are considered to 
be the way of transferring the embodiment with a substance which is then disposed of.

Reproduction health problems (anti-natal and post-natal) are not paid much attention and are considered as a built in part of the child bearing and child rearing. Termination of pregnancies are resorted in cases of pre-marital and extra-marital relationship, with the help of local herbalist without attracting much attention. In the study area, pre and extra-marital relations are of common place, but care is taken not to attract public attention. High incidence of STDs has been reported among the tribals of Rajasthan. Tribals believe that there are three different sources from which such diseases (sujak) may be contracted and none of it is remotely connected with sexual behaviour. These are namely: (i) if sakkar or gur is eaten together with the bajri roti (millet bread) immediately followed by drinking cold water; (ii) if a person passes urine at a wet fence where someone inflicted with sujak has urinated before; and (iii) sujak may be contracted from a infected person. The folk etiology of sexually transmitted diseases is in consonance with the socio-cultural conditions of the area. Here popular medical notions make it possible to maintain a convenient morality in matter of sexuality. These local medical notions help in maintaining clandestine extramarital sexual outlets and officially acclaimed standards of pre-marital and marital social fidelity. Stability of ethical norms and culture as such is reinforced. The folk etiology attributes transmission of venereal diseases to innocent sources. Tribals use charms, amulets, perform rituals to please annoyed deities, sacrifice animals and also allows free discussion of subject in the community and medical counter measures can be taken unimpeded by shame and secrecy.

\section{HEALTH CARE AMONG TRIBALS OF RAJASTHAN}

The popular domain of illness and therapeutic practices among six tribal groups under study is pluralistic and unsystemized. Since health care is a constant choice of individuals, their perception of available alternatives and their motivation to seek cure is important. The services of a practitioner are sought only after the diagnosis has been maid. The diagnosis has two types of consequences, conceptual and physiological. The process of healing is deeply embedded in culture. The conceptual consequences of sickness, diagnosis and treatment are important in understanding and managing sickness. Sickness is a fundamental assault on person and society, is a matter of deepest human concern; affecting the life and death, it can induce deep emotional arousal. The experience of healing can be highly marked. The person experiences some pain and goes to a healer for a diagnosis, who after diagnosis suggests treatment. The actual representation of metaphors for illness and cure act upon to restore harmony to the disturbed community.

The strategy a person chooses for the treatment of his or her illness or that of a relative depends on personal experiences and preferences. The tribal response to health problems reveal a multiple and simultaneous usage of home remedies and multiple therapy depending on the cultural logic based on medicine of body fluids and supernatural dimensions. The various practitioners whose services are sought are spiritist (Bhopa), traditional herbalist (jaangar/ jaankar) and public health practitioners. In Rajasthan the indigenous healers (spiritists and herbalists), though very popular lack official status as Amchi-practitioners of Tibetan medicine in Ladakh were settled upon (Bhasin, 1999) The traditional medical system of tribals is based on personalistic tradition of supernatural healers (Bhopa) and herbalists (jaangars) and their ministrations. These traditional healerdiviners operate within a religious paradigm, with no printed or written material to conform or support the tradition. It is assumed that these are sanctioned by their religion but with no proof. This system works on the individual healer's methods, reputation and performance. The indigenous medical system has continued in society's social cultural complexes through deeply rooted processes. It is a set of concepts of health and sickness that reflect certain values, traditions and beliefs based on people's way of life. It is a "constant process of conformity to contemporary psychological needs with in a recreated cultural identity"'(Wijsen and Tanner, 2001). Levi-Strauss (1967) description of the Shaman and his healing techniques shed light on the relationship between process and consequences of healing. The "Shaman provides a language (p.198) and like psychoanalyst, allows the conscious and unconscious to merge." This he achieves through a shared symbolic system and curing of one sick person improves the mental health of the group. In this context," the patient 
performs a very important social function and validates the system by calling in to play the group's sentiments and symbolic representation to have them" become embodied in real experience (pp.180-182).For these healers, the mind, the body, and the empirical field are one. Among tribals Bhopa,the ritual therapist can be understood as a healer of the mind and body as well as community. This he achieves through his status as the interpreter of symbols, those cultural instruments for perceiving and arranging reality. They are significant vectors of a force that compels mind, matter and experience (RomanucciRoss,1980b). The Bhopas are specialists possessing powers to heal or prevent illness and disaster. It is believed by the tribal that illness emanates from a disjunction of a quasi-equilibrium maintained between man, his environment and the supernatural. An individual and super individual force can disrupt the established order. The restoration of the order or the return to health can only be achieved through a healer. The different healers in the tribal society have recourse to the use of medicinal plants, animal products or minerals and therapeutic rituals. The availability of different healers enables tribals to switch from one type of health practitioner to another in search of the best. The tribal who can avail bio-medical or Ayurvedic services do so without being familiar with the theoretical principles of the medical system Though the economic status of the households differ, they show certain similarities in their behaviour in case of illness. They employ pluralistic strategies not perceiving any conflict among these alternatives, nor do they seem to perceive them as different systems, but rather as a variety of options, among which they can choose.

Most usage is sequential but some is simultaneous. For example, an infant who is being given prescribed medicine for diarrhoea, may also be-taken concurrently to a Bhopa for evil eye or given home remedies. Although certain illnesses such as evil eye are thought to be cured only by indigenous healers, this does not preclude the use of biomedicine to treat the symptoms. Gonzales (1966) reports that in Guatemalan, the symptoms are treated with bio-medicine, while the cause of illness is dealt with through a folk specialist. Traditional theories of illness etiology are often multifactorial and multilevel (i.e. immediate and ultimate levels of causation) which permits the use of different treatment resources for different causal factors and levels (Cosminsky, 1977). As reported by Cosminsky (1980) for Guatemalan plantation, pluralistic behavior among tribal population groups is pragmatic, often based on trial and error, perceived effectiveness, uncertainty of illness causation and expectation of speedy recovery. In addition to this empirical and pragmatic behavior, however, is the role played by faith in the supernatural or spiritual in curing. As a person is simultaneously a body, a self (psyche) and a social being, so are the healers of the tribals. Among tribals of Rajasthan, Bhopas attempt to resolve sickness caused by the disorder of the "social self", Jaangar on the other hand claim to cure diseases which arise from disorders of body by means of an elaborate diagnostic system and pharmacopoeia. The tribal healers accentuate different aspects of tribal personality. Social or cultural bound syndromes referred as "deity's illness" or "devta ki bimari" (illnesses such as bodily pains, possession etc. caused by the annoyed spirits, evil eye)are generally treated by ritual cleansing ceremonies (jhara ), and physical ailments by jaangar/ jaankar, through administration of herbal extracts. The characteristics of certain ailments points to the cause and mode of action accordingly. These "fixed-strategy diseases" (Beals, 1980, pp194-95) automatically affirm to particular type of treatment. Self or home treatment is usually the first step in medical care, consisting primarily of herbs, barks of trees, flowers, roots, leaves, seeds etc. Many of these are commonly known. Some grow wild in the area, some are cultivated in home gardens and others are bought from the market. Herbs are assigned a hot, cool or cold quality and are incorporated in to local hotcold classification system relevant to illness causation and treatment. The treatments are known to elders in the house or neighbourhood or suggested by local therapists. Traditional medical knowledge is coded into household cooking practices, home remedies and ill health prevention and health maintenance beliefs and routines. Treatment is generally a family based process. The advice of family members or other important members of community influences health behaviour.

It was observed from the ethnographic data that tribal use curative materials from plants either in raw form(tying leaves, smearing latex or using the plant as a tooth brush) or extracts made by squeezing or crushing the plant are used on 
wounds or bleeding parts while crushed plants are used locally as paste or concoctions of different herbs are orally administered. Tribals believe that the therapeutic use of proper diet and available medicinal herbs in the area help in restoring the general health of the community. Apart from these local healers, for a range of illnesses (fever' headache) referred as "angrezi bimari", tribal prefer to go to 'doctor'. In tribal areas there are unregistered medical doctors called "Bengali doctor" or "Gujarati doctors" and Ayurvedic vaidya who are popular among tribals. These doctors are not fully qualified but prescribe biomedicine and freely give injections to patients who lack formal education base and cannot distinguish between qualified or unqualified physicians or vaidya.

This pluralistic medical situation of doctors and deities in tribal areas provide flexibility and fulfills different needs of the community. The folk systems are open as manifested by eclecticism of both the clients and practitioners, who adopt and adapt aspects from an array of co-existing medical traditions. This openness of folk systems, as Press (1978) point out, is manifested by the acceptance of inputs from other/alternative health systems, and also inputs from institutional sectors such as religion and family. According to Landy (1974) the traditional healer role stands at the interstices religion, magic and social system and gain its power from this position. This contrasts sharply with the closeness of biomedicine, which is "discontinuous from ordinary social process" (Press, 1978; Manning and Fabrega,1973) and is unaccommodating to alternative systems.

A general quantitative survey on the utilization of multiple therapy system among tribals impart impression that they have inclination towards indigenous therapy. In the study area, out of 46 villages, two villages have primary health center, seven villages have the facility of primary health sub center, three villages have Ayurvedic dispensaries and one village has a Unani health center. These relatively new biomedical instit-utions coexist with a diversity of other health resources. The multiple medical systems available to tribals and the options available to any specific group are many. Therapies include spiritual action, religious rituals, exorcism, herbal remedies and diet to prevent future illness. Diagnostic and healing techniques such as nabj or nadu (pulse diagnosis), devala or grain diviner (are religious technicians who diagnose chronic diseases using grains) are popular. The grain diviner perform oracle by plucking grains $(a k s)$ and predict the future of the treatment. The explanatory framework of tribals fail to see conflict between biomedicine and healing rituals. Throughout their lifetime they have used the two (the ritual healing and herbal administrations) simultaneously, tribals do not find it odd to use 'angreji davai' alongside the rituals of Bhopa. It seems that among tribals biomedicine has simply added a further dimension to existing medical system. Biomedicine has not replaced the local medical traditions. As far as curative medical services are concerned, these have been embraced more easily than preventive services, as was observed in case of immunization. All groups were not ready for the immunization, as the results of the scientific curative medicine are much more easily demonstrable than the results of preventive medicine. As there are different therapies available to tribals to opt for, a sequence of resort does not appear to exist, although the trend is to begin with home remedies to bhopa to jaangar to biomedical doctor, as the course of the illness proceeds and become more serious. However, there is a back and forth movement between resources or a shorten approach, often based on referrals and advice from relatives and neighbours and other practitioners, which seems to be associated with desperation over the perceived increasing severity of an illness. When a person is sick, he or his family members are primarily interested in getting his health restored, for which they unhesitatingly combine different treatments irrespective of their ontological, epistemic, moral and aesthetic foundation. Medical pluralism results out of this orientation where attainment of health is primary objective and the individual is treated in its holistic self.

In tribal Rajasthan, there is no medicalization of folk-medicine by western medicine- the active attempt by official providers of health care to impose a standard structure on diagnostic and curing practices as discussed by RomanucciRoss of medicine in Italy (Romanucci-Ross, 1997:2). Despite penetration of biomedicine in these remote areas, traditional ideas of disease and therapies prevail. The total commitment of the believers in the traditional system persists and so does the belief of the patient in the healer, regardless of result. Traditional health culture has generally been accused of erecting barrier to the use of biomedicine and bifurcation between traditional and modern medical systems still obtains in the anthropological literature. It was believed by earlier authors that these two systems 
are discrete and biomedicine will replace traditional medical systems over time (Foster and Anderson, 1978). This study is one of many that show that traditional medical practices as well as bio-medicine co-exist. As traditional medical systems have survived in this area for such a long time, its therapeutic value and what is retainable of traditional system and how these can be upgraded through education, licensing and incorporation in to state health planning becomes important. The state health programmes are well intended but lack anthropological consultation. As a consequence, the traditional medicinal care practiced in the area having both herbal and ritual form of curing is not considered important . The cultural importance of ritual care and role of medicinal plants (their properties as they relate to healing, their symbolic values and their procurement from environment) in the traditional medical system of tribal is of great value. There are large number of illnesses where oral application of herbal preparations are a frequent part of treatment (e.g. fever, dysentery, diarrhoea, malaria, cough and cold, eye-ailments, Guinea worm (nadu), stomach-ache etc.). However at some point the tribal see the limits of such phytotherapeutic forms of treatment. Any illness that is associated with severe pain, long lasting and not responding to herbal treatment requires the help of supernatural. Tribal relate their ritual needs to supernatural powers and ask for help and forgiveness. The state sponsored medical system do not look at "indigenous medicine" as whole and fail to see the sociocultural basis of its uses .

The main strength of the Traditional Medical System (TMS) of tribal is its capacity to stand as psycho support system. The explanatory model of TMS greatly emphasize the notion of disharmony as a cause due to man's relations with the supernatural powers and other bodily related illnesses caused by drinking and eating wrong things. Transferability and transgressionality of supernatural wrath to the members of the society makes it a powerful force in social control.

KEY WORDS Tribes; ethnographic data; biomedicine; North India.
ABSTRACT This paper examines the medical systems utitilized by the tribals of Rajasthan. Local medical traditions have continued to co-exist with biomedicine among tribals of this semi-arid-zone. An attempt has been made to focus on health, sickness and therapy form the point of view of ordinary tribals of Rajasthan, real or prospective patients and their family members as well as local practitioners.

\section{REFERENCES}

Beals, A. 1980. Strategies of resort to curers in South India. in Asian Medical Systems. C. Leslie, (ed.). Berkely: University of California Press

Bhasin,V. 1997. "Medical Pluralism and Health Services in Ladakh." Journal of Social Sciences, 1: 43-69.

Cosminsky, S. 1977. "Impact of methods on the analysis of illness concept in a Guatemala community". Social Science and Medicine. 11: 325 (1977).

Cosminsky, S. 1980. "Medical pluralism on a Guatemala Plantation." Social Science and Medicine, 14B: 267278

Foster, G.M. and B.G. Anderson. 1978. Medical Anthropology. New York: John Wiley and Sons.

Frake, D. 1961. "The diagnosis of disease among the Subaru of Mindanas." Americn Anthropologist, 113132.

Geertz, C. 1973. The Interpretation of Culture. New York: Basic Books.

Gonzales, N.S. 1966. "Health behavior in cross-cultural perspective: A Guatenmala example." Human Organization, 25: 122.

Kleinman. A.P. 1980. Patients and Healers in the Context of Culture: An Exploration of the Borderland Between Anthropology, Medicine and Psychiatry. Berkely: University California Press.

Landy, D. 1974. "Traditional Curers Under the Impact of Western Medicine." American Ethnology, 1: 103 (1974)

Levi-Strauss, C. 1967. Structural Anthropology. New York: Doubleday.

Manning, P. and H. Fabrega Jr. 1973. "The experiences of self and body: Health and illness in Chiapas highlands." in Phenomenological Sociology. Psathas, (ed.), pp 290. New York: Wiley.

Marriott, M.1990. "Consructing an Indian ethnosociology." in India Through Hindu Categories M. Marriott (ed.). pp 1-39. New Delhi: Sage.

Romanucci-Ross. 1980. "On the researching of lost images." Anthropology and Humanism Quaterly, 5 (1): $14-20$

Romanucci-Ross. 1997. "Creativity in illness: Methodology, linkages to the logic and language of science in folk pursuit of health in Central Italy." in The Anthropology of Medicine from Culture to Method. Lola Romanucci-Ross, Daniel E. Moerman, and Laurence R. Tancredi (eds.) Connecticut: Bergin \& Garvey.

Wijsen, F. and R.E.S. Tanner. 2001. The Recreation of Cultural Identity. In Press.

Author's Address: Veena Bhasin, Department of Anthropology, University of Delhi, Delhi 110007 , India 